\title{
A BEHAVIOR ANNEX FOR AADL USING THE DEVS FORMALISM
}

Ehsan M. Ahmad

College of Computing and Informatics

Saudi Electronic University

Riyadh, Kingdom of Saudi Arabia

e.ahmad@seu.edu.sa
Hessam Sarjoughian

\begin{abstract}
It is essential to use modeling methods for specifying real-time and safety-critical specifications and executions as a set of computational and physical components. Thus, frameworks supporting modular, hierarchical specifications at multiple levels of abstraction for software-intensive systems are needed. Models for the structures, behaviors, and relationships benefit from separating and combining external properties and internal operations of components. Together, the Architecture Analysis and Design Language (AADL) and Discrete-Event System Specification (DEVS) provide an integrated framework where the structure and behavior designs can be systematically developed and evaluated. Needs including latency and safety analyses are supported by AADL whereas behavioral verification and validation can be supported with DEVS simulation. To create the proposed AADL-DEVS framework, a DEVS behavioral annex targeted for the DEVS-Suite simulator is developed and introduced to OSATE which supports AADL. The DEVS Annex language is detailed and exemplified using an infant incubator known as the Isolette system.
\end{abstract}

Keywords: AADL, DEVS Annex, DEVS-Suite, Safety-Critical Systems, OSATE.

\section{INTRODUCTION}

There is a growing class of systems known as Systems-of-Systems (SoS) or Cyber-Physical Systems (CPS) (Sztipanovits, Bapty, Koutsoukos, Lattmann, Neema, and Jackson 2018). These systems are challenging to build, in part, because a variety of concepts, methods, frameworks, and tools are needed to tackle their multifacetted nature (Feiler and Gluch 2012) (Zeigler and Sarjoughian 2017). Although software engineering and model \& simulation are essential, using them together is challenging, particularly due to increasing system complexity and scale. There are conceptual gaps in connecting, for example, across multiple specification abstractions. For example, it can be beneficial to employ the Architecture Analysis and Design Language (AADL) (Feiler and Gluch 2012) and Discrete Event System Specification (DEVS) (Zeigler, Praehofer, and Kim 2000) to support specifications that cover and integrate high-level architectural and low-level design specifications. That is, a key to the development of the software-intensive system is to have specifications that precisely represent decisions spanning both coarse-grain and fine-grain design needs. The former generally characterizes system's architecture (i.e., parts and their relationship). The latter primarily are focused on specifying behaviors that conform to the defined system architecture specifications. This two-step process belonging to the full life-cycle development involves combining structures and behaviors at multiple levels of abstraction. A prominent approach is known as the Model-Driven Architecture (MDA) with the Eclipse 
Modeling Framework (EMF) are increasingly used. Together, these are proving to be quite important for developing frameworks and tools for real-time, safety-critical systems.

The above serves as our motivation which is derived from the observations that any system's complexity and scale traits can be managed through dividing a system into overlapping structural and behavioral specifications across multiple levels of abstraction. In this respect, the AADL and DEVS have shown to be expressive and practical given their respective development frameworks and tools align with the MDA and Model-Based Design (MBD) in particular. In this paper, we present the DEVS Annex (DA) for complementing AADL with behavior modeling and simulation which is developed using the Open Source Architecture Tool Environment (OSATE) (Software Engineering Institute 2018) and the DEVS-Suite simulator (ACIMS 2019). The DA is a part of an ongoing research effort on an AADL-DEVS framework and tool supporting discrete-event behavioral modeling and simulation.

\section{BACKGROUND}

This section presents an overview of the Architecture Analysis and Design Language (AADL) and the Discrete-Event System Specification (DEVS) formalism. The former focuses on safety critical system architecture grounded in an integrated software and hardware abstraction framework. The latter offers a theoretical basis for specifying models that can lend themselves to virtual execution (i.e., simulation).

\subsection{Architecture Analysis and Design Language}

The Architecture Analysis and Design Language (AADL) is a declarative language for specifying system specifications (Feiler and Gluch 2012, Software Engineering Institute 2018). The AADL specification is an SAE Standard and is targeted for safety-critical embedded systems (SAE International 2017). It supports partitioning a system's architecture into modular integrable software and hardware components. The AADL has been successfully demonstrated for several industrial case studies in domains like medical and aerospace engineering. The AADL as a framework can aid specification and evaluation of run-time observable (e.g., performance and security) as well as non-run-time observable (e.g., integrability, reusability) system architecture quality attributes (Bass, Clements, and Kazman 2013). The basic concept is to specify a system's architecture as an assembly of software components (i.e., logical functions and relationships) mapped onto hardware components (i.e., an execution platform). Every software and hardware component is defined to have inputs and outputs that can be connected together to specify their integrated system-level operations and interactions. The specification language affords to declare tasks, operational modes, fault-tolerant configurations, and timing aspects of (logical or real-time) systems.

The AADL is defined in terms of software, hardware, and hybrid categories (Software Engineering Institute 2018, Feiler and Gluch 2012). The software components are classified as data, subprogram, thread, and process. The data defines characteristics such as data size. The subprogram defines a block of code with parameters. The thread defines an entity that executes an instruction flow with timing constraints (e.g., deadline) and execution regime (e.g., event-triggered). The process specifies an address space that contains the data and the thread components. The hardware components are classified as device, processor, bus, memory, virtual bus, and virtual processor. These components specify the physical properties of embedded system hardware. The device specifies things such as sensors and actuators. The processor specifies a processing unit responsible for executing subprograms. The bus defines physical communication connectors such as Ethernet and wifi between hardware components. The memory specifies physical memory such as cache. The virtual bus specifies the logical connection (protocol) between software components. The virtual process specifies software representation of hardware. The hybrid components are classified as abstract and system components. The former is for the components whose categories can be defined during architecture 
specification evolution. An abstract component can be an abstract subcomponent. Each of the software, hardware, and hybrid components has a type and multiple implementations. The system component combines and configures the components that are needed for system implementation. The detailed specifications are required for latency and safety analyses. For behavioral modeling and simulation, the AADL can be augmented with behavioral languages. Examples are the Behavioral Language for Embedded Systems with Software (BLESS) Annex (Larson, Chalin, and Hatcliff 2013) and Hybrid Annex (Ahmad, Larson, Barrett, Zhan, and Dong 2014).

\subsection{System-Theoretic Discrete-Event Simulation}

The Discrete Event System Specification (DEVS) is generally considered suitable for modeling and simulating systems (Zeigler, Praehofer, and Kim 2000). Models can communicate arbitrary data types with arbitrary timing and data/event handling. Moreover, DEVS can be used to describe any discrete event systems (Zeigler, Praehofer, and Kim 2000). For example, software (Ferayorni and Sarjoughian 2007) and mixed software/hardware systems (Muqsith, Sarjoughian, Huang, and Yau 2011) have been modeled and simulated using the Parallel DEVS formalism (Chow and Zeigler 1994) and the DEVS-Suite simulator (ACIMS 2019). Continuous models can also be specified using variants of this modeling approach. As a mathematical formalism, it lends itself for specifying structures and behaviors of Systems of Systems (SoS) including Cyber-Physical Systems (CPS).

The DEVS formalism is defined in terms of two types of modular models. One is called atomic model and another coupled model. The former type specifies the structure and behavior of elementary (basic) components that can be composed with other atomic or coupled models to specify strict hierarchical models. Every coupled model has a strict tree structure such that all of its leaf nodes are atomic models with all remaining nodes being coupled models. The DEVS formalism has a corresponding abstract simulation algorithm that has atomic and coupled simulators corresponding to the atomic and coupled models. The simulators execute the time-based dynamics of atomic and coupled models. The simulation algorithms have been realized using centralized, distributed, and parallel simulation engines (Zeigler and Sarjoughian 2017).

A parallel DEVS atomic model is a mathematical structure. It has input and output ports with values (i.e., primitive or compound messages) that specify the exterior structure of an atomic model. The internal behavior of an atomic model is specified in term of a set of state variables and a set of functions. A model can have autonomous and reactive behaviors specified in terms of an internal transition function and an external transition function. The output function allows generating output messages for any number of output ports. The time advance function captures the timing of state transitions. The confluent function can be used for specifying simultaneous internal and external events. An atomic model can have multiple input and/or output messages. The elapsed time $e$ has the role of allowing external inputs to arrive at arbitrary time instances. The $t a(s)=0$ allows instantaneous state change which is a basic capability for modeling concurrent and distributed software/hardware systems.

A parallel DEVS coupled model is also defined as a mathematical structure. Any coupled model has one or more atomic and/or coupled models. The structural specification of a coupled model includes input \& output ports, a set of components, and component coupling information. DEVS can ensure semantically identical input/output interfaces for atomic and coupled models. The coupling information is categorized as (1) the external input coupling (EIC) - coupling of coupled model input ports to input ports of some component, (2) the external output coupling (EOC) - coupling of component output ports to the coupled model output ports, and (3) the internal coupling (IC) - coupling of component output ports to input ports of components. A coupled model's behavior is, in part, due to the message exchanges between itself and its components as well as message exchanges among the components. DEVS enjoys the closed under coupling property which is key for hierarchical models. 


\section{RELATED WORK}

The existing research that relate to this work can be viewed in two perspectives. First, extending the AADL architectural core language with the DEVS behavioral modeling language. Second, the development of an integrated framework where structural and behavioral designs are supported in as much as possible under a unified specification and execution framework. In view of the first perspective, the annex mechanism in AADL is used, for example, to develop the BLESS and Hybrid (HA) annexes (Larson, Chalin, and Hatcliff 2013, Ahmad, Larson, Barrett, Zhan, and Dong 2014). The BLESS annex uses states and state transitions for specification and proving the correctness of the discrete behavior of control systems while the HA supports modeling the monitored and controlled signals of continuous physical systems. From the second perspective, early works have utilized meta-programmable and model-integrated computing for virtual systems-of-systems evaluations (Sztipanovits and Karsai 1997). DEVS simulation is also used with AADL for verification of TT-Ethernet modeled using AADL (Robati, El Kouhen, Gherbi, and Mullins 2015).

Considering the DEVS modeling approach, it is advocated to be used for aviation system simulation by combining it AADL and the Aviation Scenario Definition Language (ASDL) (Jafer, Zeigler, and Kim 2018). The DEVSML (Mittal and Douglass 2012), EMF-DEVS (Sarjoughian and Markid 2012), and DEVS Natural Language (DNL) (Zeigler and Sarjoughian 2017), among others, have been developed for the Parallel DEVS formalism. The DEVSML and DNL use Xtext which supports the Extended BNF grammar within the Eclipse Modeling Framework (EMF). The EMF-DEVS uses the Ecore language also supported by EMF. Other research include automation for translating Conceptual DEVS Models to code for target simulators (Cristiá, Hollmann, and Frydman 2019). Compared to these approaches, the proposed DA grammar is grounded in both the AADL and DEVS modeling languages. Furthermore, the time constraints defined for the AADL-compliant model can be used to extend the DEVS Annex grammar to support Action-Level, Real-Time DEVS (ALRT-DEVS) (Sarjoughian and Gholami 2015, Gholami and Sarjoughian 2017) modeling formalism. Such AADL-DEVS models can execute under real-time constraints defined in the model and enforced by the host the DEVS-Suite simulator's host computing platform. A first prototype of the DA supporting logical-time simulation using the DEVS-Suite simulator is developed as a plug-in for OSATE. The DA provides a basis for combined static and dynamic design, verification, and validation.

\section{A RUNNING EXAMPLE}

In order to demonstrate an application of the proposed behavior specification language for complex hierarchical safety-critical systems, this section presents a case study of the Isolette system, an infant incubator described in the Requirement Engineering Management Handbook (REMH) published by Federal Aviation Administration (FAA) in 2009 (Lempia and Miller 2009). This specification is rich enough to highlight the need for our proposed annex. We use it to show the application of the DEVS Annex notation for modeling system behavior, and simulation using DEVS-Suite.

Figure 1(a) depicts the operational context of the Isolette system to maintain the temperature of the Air within the desired range set by the Nurse using Operator Interface. The Thermostat monitors the Air temperature through the Temperature Sensor and attempts to manipulate it with the Heat Source actuator. The control strategy followed by the Thermostat is derived from a process model that is implicit in the interpretations it gives to the current Air information coming from the sensor, and the commands it has issued to the actuator. In this study, we focus on modeling and simulation of the behavior of the Thermostat, and its interactions with the Heat Source and Temperature Sensor units. The internals of the Operator Interface and continuous behavior of the Air are not considered. To keep the current temperature within the desired range, Thermostat performs two major functions; i) Monitor Temperature, and ii) Regulate Temperature. 


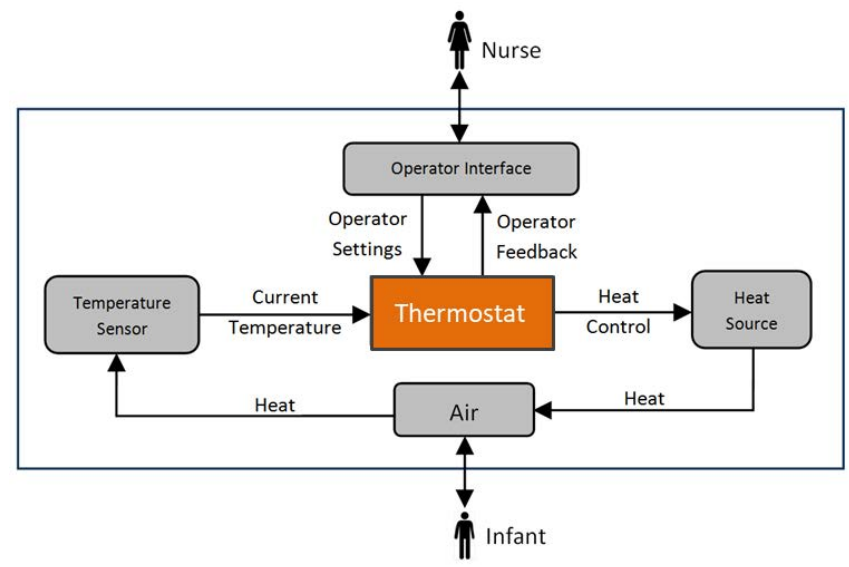

(a) Isolette context diagram

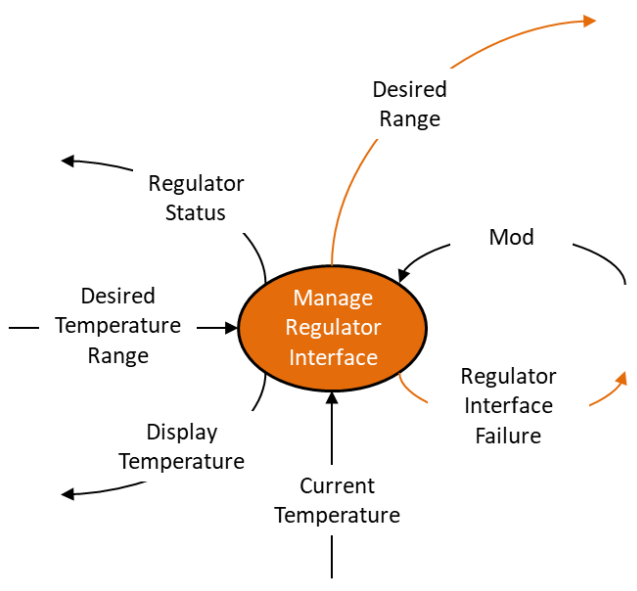

(b) Manage regulator interface function

Figure 1: Operational context of Isolette system with a subfunction of the Thermostat.

Figure 1(b) depicts the Manage Regulator Interface function which is one of the four subfunctions performed for temperature regulation. Manage Regulator Interface function obtains Desired Temperature Range from Operator Interface and Mod from Manage Regulator Mode subfunction (not shown here) and reports back the Regulator Status, Display Temperature, Regulator Interface Failure, and Desired Range according to a well-defined set of requirements. Below we describe the functional requirements of the variables Regulator Interface Failure, and Desire Range to be considered in this paper;

- REQ-MRI-6: If the Status attribute of the Lower Desired Temperature or the Upper Desired Temperature is Invalid, the Regulator Interface Failure shall be set to True.

- REQ-MRI-7: If the Status attribute of the Lower Desired Temperature and the Upper Desired Temperature is Valid, the Regulator Interface Failure shall be set to False.

- REQ-MRI-8: If the Regulator Interface Failure is False, the Desired Range shall be set to the Desired Temperature Range.

- REQ-MRI-9: If the Regulator Interface Failure is True, the Desired Range is UNSPECIFIED.

Modeling the just described behavior of the Isolette example with the proposed DEVS Annex is detailed in Section 5 along with an AADL implementation while the type classifier is first presented here. As specified in REQ-MRI-8 and REQ-MRI-9, variable Desired Range is directly related to variable Regulator Interface Failure, they are modeled using a single AADL thread manage_interfaceFailure_desiredRange component. A complete AADL model of the Regulator Temperature functionality with detailed behavior specified using DEVS Annex and corresponding Java classes for the DEVS-Suite simulator are automatically generated using our ADDL to DEVS Code Generation Engine (ADCoDE) tool https://www.github.com/ehah/ ADVENT.

The type classifier of Listing 1 declares the interface of this thread component. Due to space limitation, only two (one in and one out) ports are shown in Listing 1. The in data port lower_desired_temperature is used to get the lower value of the desired temperature. The out data port unspecified_temp is defined to transmit the string UNSPECIFIED as per requirement REQ-MRI-9 and is modeled as unspecified_value.

In the properties section, thread manage_interfaceFailure_desiredRange is declared as periodic with a period of $100 \mathrm{~ms}$. 
Listing 1: Manage Interface Failure and Desired Range component type.

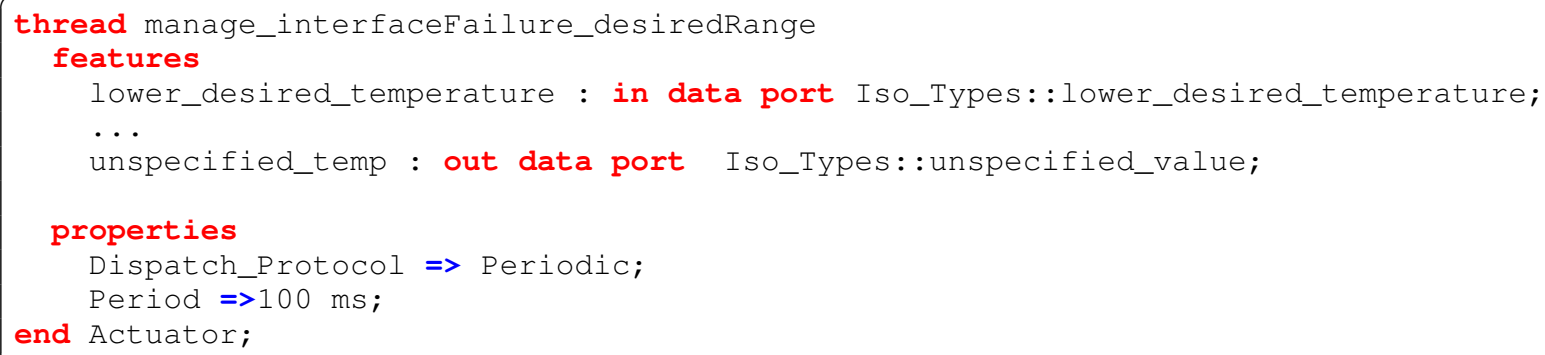

\section{DEVS ANNEX}

This section presents the constructs of the DEVS Annex (DA), an extension to AADL for discrete-event modeling. Each DA section is described in detail with its syntax, and grammar with appropriate examples. The annex subclause grammar and semantics are based on the DEVS formalism. This annex brings with it the ability to model detailed behaviors at the component level based on the concept of the Atomic DEVS and at system level based on the concept of the Coupled DEVS.

The rest of this section contains the Extended Backus-Naur Form (EBNF) of the DA grammar, in which: literals are printed in bold; alternatives are separated by a pipe |; groupings are enclosed with parentheses ( ); square braces [ ] delimit optional elements; and the closures \{\}$+$ and \{\}$^{*}$ are used to signify one-or-more, and zero-or-more of the enclosed element, respectively. The entities, variables, states, and behavior are the sections of a DA subclause, each of which is dedicated to specify particular aspect of a detailed behavior model. Following is the grammar of the DA subclause:

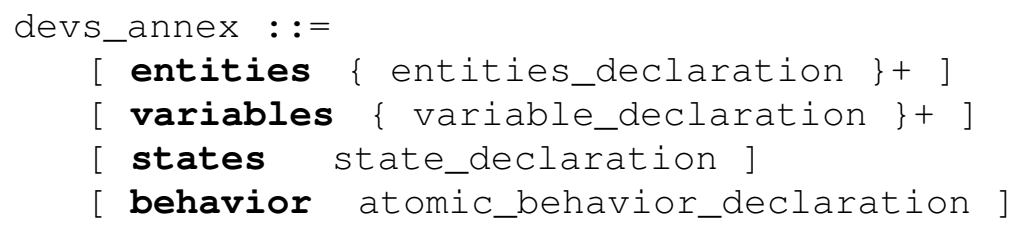

Entities section: Following is the grammar of the entities section. The italicized prefix for identifiers gives "hints" about the kind of identifier.

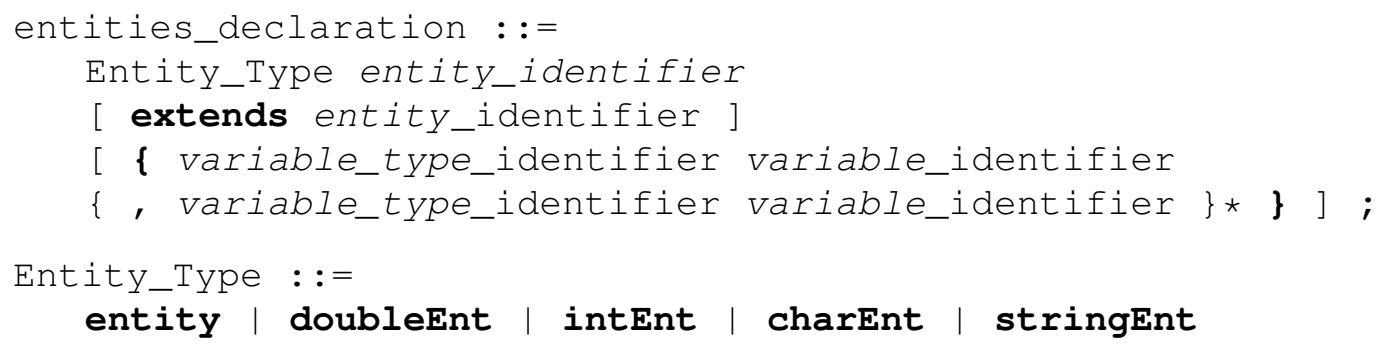

Variables section: Local variables in the scope of a DA subclause are declared in the variables section along with their data types. Data types are assigned to variables by classifier references to the appropriate AADL (user definable) data components. Each variable must have an initial value specified after $=>$. Depending on the data type, an initial value can either be primitive or compound consisting of more than one primitive values separated by commas and enclosed in parenthesis. Following is the grammar of the variables section: 


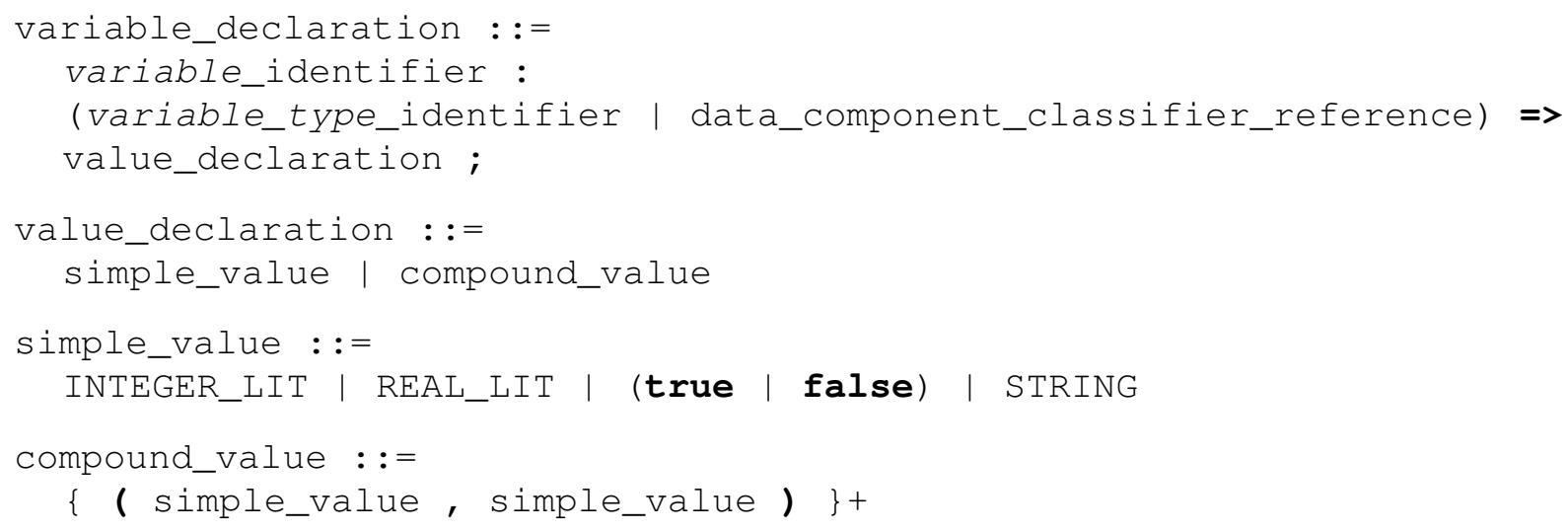

The referred external data component must either be part of the package containing the component being annotated, or declared within the scope of another package (i.e., imported using the AADL with clause).

The variables section in Listing 2 shows the variable declarations of the DA specification for the example Isolette system. They have been identified using the above four system requirements and the variable udt representing the variable Upper Desired Temperature. The data types lower_desired_temperature and upper_desired_temperature specified as ldt and udt are the same as those explained in Section 4.

The DA sublanguage is designed to specify behavior that can be simulated using DEVS-Suite simulator. The core of DEVS-Suite has also been extended with new classes to incorporate with AADL structure elements and data modeling. The ADCoDE has been developed as part of the AADL-DEVS framework to partially translate DEVS Annex specification to counterpart DEVS-Suite code. The IntegerRange class is introduced to represent an integer range with three variables; minVal, maxVal, and $c$ Val representing the minimum, maximum, and current values, respectively. For initialization, an IntegerRange object only requires a value for $c \mathrm{Val}$ while the values for the other two variables are automatically extracted from respective models during code generation. Thus, both the $1 \mathrm{dt}$ and udt variables are initialized with the value (0, "Invalid"), where the 0 is the initial value for the first element which is to be specified as an IntegerRange, and "Invalid" is the value is for the second element to be specified as enumeration. Details of the DEVS-Suite extension and the ADCoDE are out of the scope for this paper and they are planned to be presented in future works. The variable rif represents either true or false indicating the validity of the Lower and Upper Desired Temperature values. The variable pd is of the type thread_period and is set to $100 \mathrm{~ms}$.

States section: Behavior in a DA subclause is modeled as a state-transition system starting from the initial state and continue following the internal and external transitions. All the admissible states of a particular component are defined in the states section. Each state specification has a name followed by the time advance function $(t a)$ stating the time to remain in a particular state before the next transition. States with $t a=0.0$ allow instantaneous transitions to the new state while states with $t a=$ INFINITY model the final state. DA only allows one starting state labeled as initial while the unlabeled states are considered as transient states. A complete behavior starts from an initial state, suspends in transient states (with different $t a$ values) until the next internal or external transition while performing actions upon each transition, and ends in the final state. The grammar of the states section is as follows:

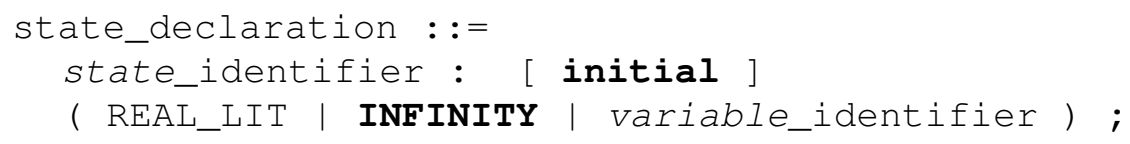

The states section of Listing 2 contains declarations for admissible states of our running example. The start state marked as initial with $t a=0.0$ represents an instantaneous starting state. The state 


\section{Ahmad and Sarjoughian}

Chk_Status with $t a=\mathrm{pd}$ is a transient state. The validity of the status element of the variables ldt and udt is checked in this state and the variable rif is set accordingly. Th state Set_Vars is also an instantaneous state with $t a=0.0$. It has been defined to facilitate output generation as these are only allowed on internal state transitions.

Listing 2: Manage Interface Failure and Desired Range component implementation.

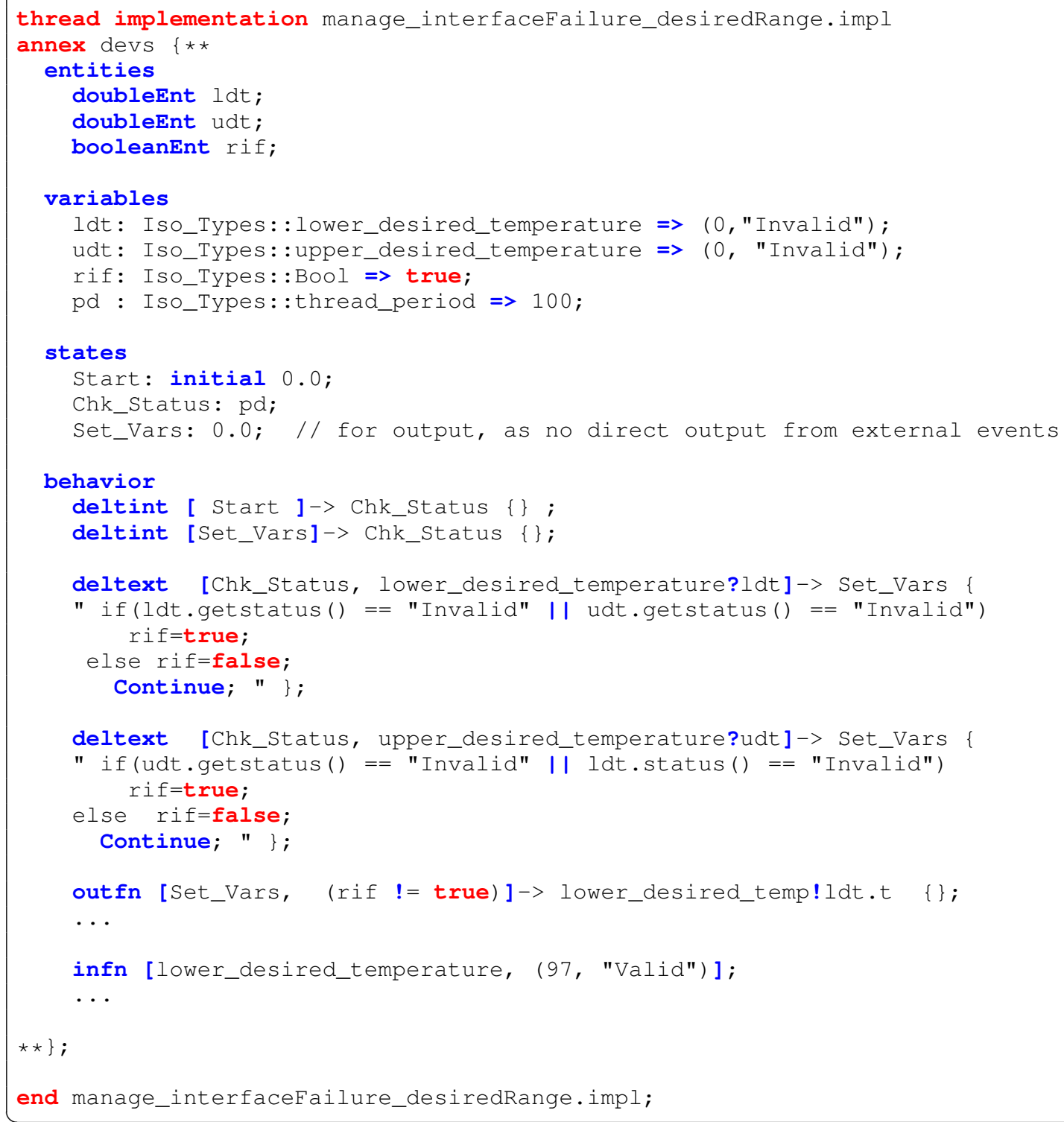


Behavior Section: The behavior section of a DA subclause is used to specify the behavior of an AADL component in terms of four functions. The functions deltext and deltint are used to specify the external and internal transitions, respectively. The function outfn specifies the system's output based on particular internal or external events and the test inputs are specified using the infn functions.

The grammar of the behavior section is as follows.

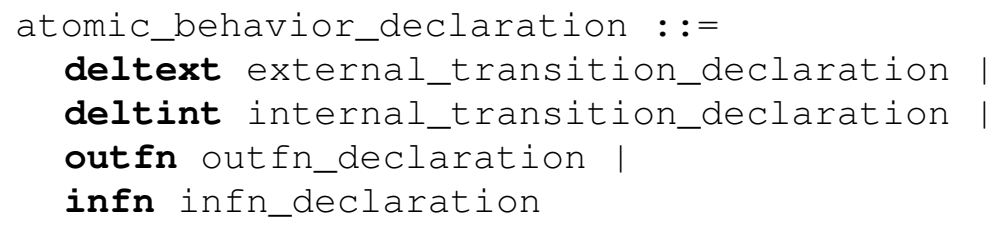

Below, we explain each of these functions which may constitute a component's behavior individually or in combination with other functions.

External Transition Function: The deltext function is used to model external transitions from one source state to a target state caused by input messages. System's response to external messages is specified as behavior action and depends on the current state, specific inputs, and the time elapsed in the current state.

An external message in a DA subclause is composed of the respective port name, the value received, and a variable to store this value for future local use. It is important to note that type of the variable must match with data classifier specified along with the port in the type classifier. Behavior action in DA specifies the activities is performed upon completion of the external transition. The current version of the DA sublanguage uses a string to model these actions and should contain syntactically correct Java language statements. Extension of the sublanguage to allow common Java constructs is an important future work in this direction. The grammar for the deltext is provided below.

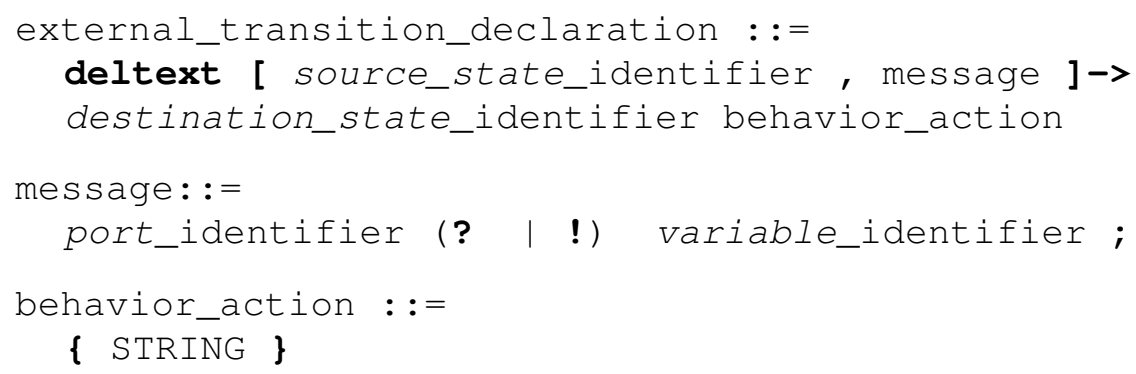

In Listing 2, the external transition functions specified in line 24 and line 30 have chk_Status as the source state and set_Vars as the destination state. The external message in the first function specifies that the message received on the port lower_desired_temperature is stored in the variable ldt while in the second function the message received on the port upper_desired_temperature is stored in the variable udt. The behavior actions contain an if-else statement for setting the value for the rif variable. If the status of the ldt or udt variable is Invalid, then the variable rif is assigned true; otherwise it is assigned false as per requirements REQ-MRI-6 and REQ-MRI-7. The continue statement is used to specify the elapsed time.

Internal Transition Function: The deltint function is used to model state transitions defined by two state values and $t a$. Target state identifier follows the source state identifier and is followed by the behavior action specification. As mentioned earlier, the current version of the sublanguage uses a string to model these 
actions and should contain syntactically correct Java language statements. The grammar for the deltint function is provided below.

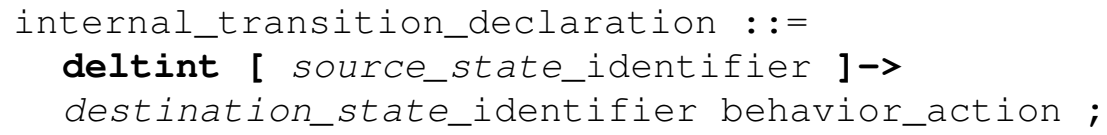

For our running example, two internal transition functions are defined in Listing 2. The internal transition function specified in line 21 has start as a source state and Chk_Status as a destination state. The internal transition function specified in line 22 has set_Vars as a source state and Chk_Status as a destination state. The empty braces indicate that is no behavior action.

Output Function: The out fn function is used to model observable outputs given state information. Output messages are only generated on the internal state transitions. If an output is required to be generated on an external transition, a state transition with $t a=0.0$ is needed. An output message contains the out port name followed by ! sign and the data to be transmitted. An out fn specification may also contain conditional expression to further restrict output generation. A conditional expression is a boolean expression that may have relational operators while the numeric expressions are defined to specify the arithmetic operations $(+$, -, * $/(\mathrm{mod})$ and the power operation $(\wedge)$. The grammar for the out fn function is defined below.

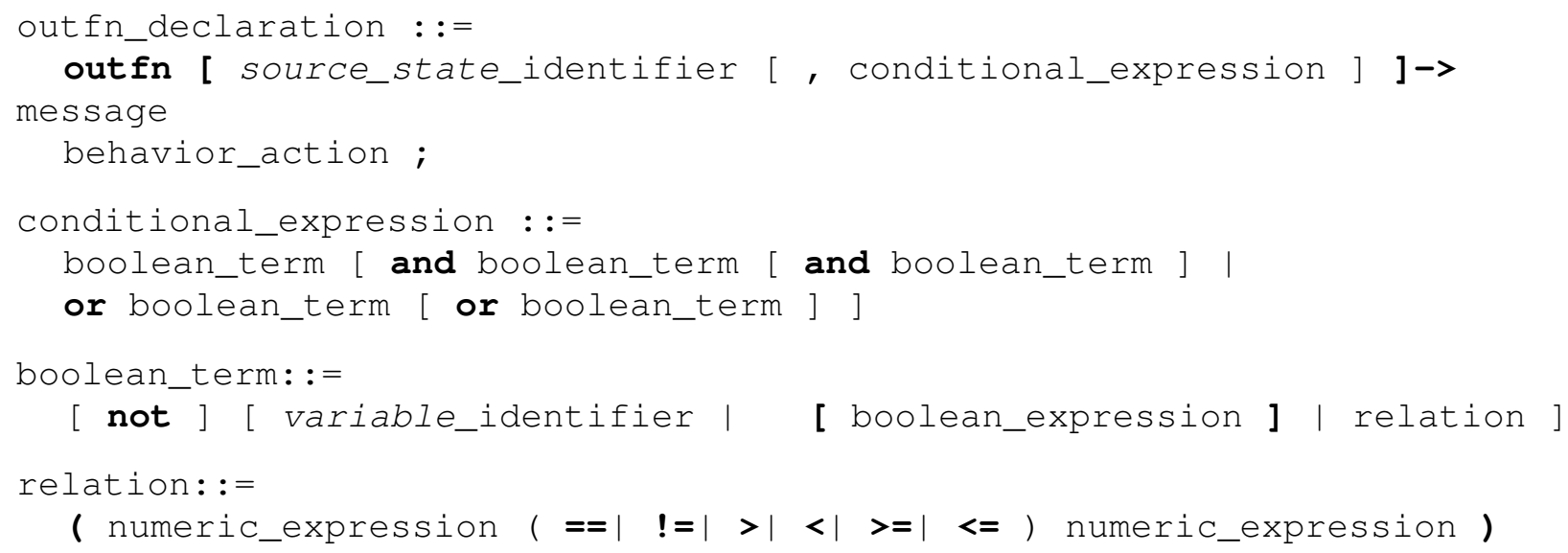

For our running example, four output functions are required to be specified but due to space limitation only one output function in line 36 is shown in Listing 2. It has Set_Vars as the source state and (rif $!=$ true) as the guard condition. Following the requirement REQ-MRI-8, this output function specifies that the temperature value is only transmitted through the out port lower_desired_temp if the guard condition holds.

Test Input Function: The infn is used for stepping the simulation using one input at a time. This function is not a part of the DEVS formalism rather used by the DEVS-Suite (the target simulator). The specification for this function contains one or more input port names followed by their assigned input values. Primitive values are specified directly and compound values are parenthesized while separating and ordering the data items with a comma, .

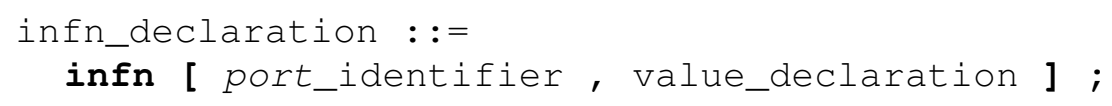

For our running example, in Listing 2, input function in lines 39 is specified to test for lower_desired_temperature in port with values (97, "Valid"). The first element in this compound 
value represents the current temperature while the second element represents the status of the current temperature.

\section{CONCLUSION AND FUTURE WORK}

The need for software-centric systems can be satisfied in part using frameworks that simplify model development and simulation. The MDA and MBD concepts, frameworks, and tools provide a basis for developing computational platforms that conform to the syntax and semantics of the AADL and DEVS. In this research, we have proposed and developed a DEVS Annex such that it conforms in an integrated fashion to both the AADL and DEVS modeling approaches. The combined use of AADL and DEVS supports structural latency/safety static analyses and behavioral dynamic simulation. These approaches share a foundational common modular, hierarchical declarative model specifications with one supported with the OSATE and DEVS-Suite development and execution environments. A prototype of the DEVS Annex is introduced into the OSATE framework. A prototype transformation engine is also developed for converting the OSATE DEVS models to their counterparts for the DEVS-Suite simulator. The AADL-DEVS as an integrated framework is proposed to serve as a methodology for disciplined modeling spanning from architecture to design specifications. The AADL specifications supporting latency and safety analyses are extended with DEVS specifications supporting logical-time simulations. The AADL-DEVS framework is used to demonstrate the applicability of the integrated AADL and DEVS model for a real-time, safely-critical infant incubator (Isolette) system. Future research includes further development and integration of the DEVS Annex with its DEVS-Suite simulator transformation engine to be a plugin for the OSATE tool. Another research direction is to extend the DEVS Annex with ALRT-DEVS modeling and simulation. Other closely related future research is to support DEVS Annex behavior modeling with the UML Statecharts and Activity specifications.

\section{REFERENCES}

ACIMS 2019. "DEVS-Suite Simulator, Version 5.0.0”. https://acims.asu.edu/software/devs-suite.

Ahmad, E., B. R. Larson, S. C. Barrett, N. Zhan, and Y. Dong. 2014, October. "Hybrid Annex: An AADL Extension for Continuous Behavior and Cyber-physical Interaction Modeling”. Ada Lett. vol. 34 (3), pp. 29-38.

Bass, L., P. Clements, and R. Kazman. 2013. Software Architecture in Practice. 3rd ed. Addison Wesley, SEI Series in Software Engineering.

Chow, A., and B. Zeigler. 1994. "Parallel DEVS: A parallel, hierarchical, modular, modeling formalism". In Proceedings of the 26th conference on Winter simulation, pp. 716-722. Society for Computer Simulation International.

Cristiá, M., D. A. Hollmann, and C. S. Frydman. 2019. “A multi-target compiler for CML-DEVS”. Simulation vol. 95 (1).

Feiler, P. H., and D. P. Gluch. 2012. Model-Based Systems Engineering. 1st ed. Addison-Wesley Professional.

Ferayorni, A. E., and H. S. Sarjoughian. 2007. “Domain Driven Simulation Modeling for Software Design”. In Proceedings of the 2007 Summer Computer Simulation Conference, SCSC '07, pp. 297-304. San Diego, CA, USA, Society for Computer Simulation International.

Gholami, S., and H. S. Sarjoughian. 2017. "Action-Level Real-Time Network-on-Chip Modeling”. Simulation Modelling Practice and Theory vol. 77, pp. 272-291.

Jafer, S., B. Zeigler, and D. D. H. Kim. 2018. "A Framework for Rapid Configuration of Collaborative Aviation System-of-Systems Simulations". In Modelling and Simulation for Autonomous Systems, edited by J. Mazal, pp. 92-105. 
Larson, B. R., P. Chalin, and J. Hatcliff. 2013. "BLESS: Formal Specification and Verification of Behaviors for Embedded Systems with Software". In NASA Formal Methods, 5th International Symposium, NFM 2013, Moffett Field, CA, USA, pp. 276-290.

Lempia, D. L., and S. P. Miller. 2009. "Requirement Engineering Management Handbook". Technical Report DOT/FAA/AR-08/32, Federal Aviation Administration.

SAE International 2017. “AADL AS5506C”. https://www.sae.org/standards/content/as5506c/. [Online; accessed 10-January-2019].

Software Engineering Institute 2018. "Architecture Analysis and Design Language". http://www.aadl.info/ aadl/currentsite/. [Online; accessed 10-October-2018].

Mittal, S., and S. A. Douglass. 2012. "DEVSML 2.0: the language and the stack". In 2012 Spring Simulation Multiconference, SpringSim '12, Orlando, FL, USA, March 26-29, 2012, Proceedings of the 2012 Symposium on Theory of Modeling and Simulation - DEVS Integrative M\&S Symposium, pp. 17.

Muqsith, M. A., H. S. Sarjoughian, D. Huang, and S. S. Yau. 2011. "Simulating adaptive service-oriented software systems". Simulation vol. 87 (11), pp. 915-931.

Robati, T., A. El Kouhen, A. Gherbi, and J. Mullins. 2015. "Simulation-Based Verification of Avionic Systems Deployed on IMA Architectures". In MoDELS'15.

Sarjoughian, H. S., and S. Gholami. 2015. "Action-level real-time DEVS modeling and simulation". Simulation vol. 91 (10), pp. 869-887.

Sarjoughian, H. S., and A. M. Markid. 2012. "EMF-DEVS modeling". In 2012 Spring Simulation Multiconference, SpringSim '12, Orlando, FL, USA, March 26-29, 2012, Proceedings of the 2012 Symposium on Theory of Modeling and Simulation - DEVS Integrative M\&S Symposium, pp. 19.

Sztipanovits, J., T. Bapty, X. D. Koutsoukos, Z. Lattmann, S. Neema, and E. K. Jackson. 2018. "Model and Tool Integration Platforms for Cyber-Physical System Design". Proceedings of the IEEE vol. 106 (9), pp. 1501-1526.

Sztipanovits, J., and G. Karsai. 1997. “Model-Integrated Computing”. IEEE Computer vol. 30 (4), pp. 110111.

Zeigler, B. P., H. Praehofer, and T. G. Kim. 2000. Theory of Modeling and Simulation. 2nd ed. Orlando, FL, USA, Academic Press, Inc.

Zeigler, B. P., and H. S. Sarjoughian. 2017. Guide to Modeling and Simulation of Systems of Systems. 2nd ed. Simulation Foundations, Methods and Applications. Springer.

\section{AUTHOR BIOGRAPHIES}

EHSAN M. AHMAD is an Assistant Professor in the College of Computing and Informatics at Saudi Electronic University. He holds a Ph.D in Computer Science and Information Technology. His research interests include system architecture analysis, program verification, discrete-event simulation, and computational thinking. His email address is e.ahmad@seu.edu.sa.

HESSAM S. SARJOUGHIAN is an Associate Professor of Computer Science and Computer Engineering in the School of Computing, Informatics, and Decision Systems Engineering (CIDSE) at Arizona State University (ASU), Tempe, AZ, and the co-director of the Arizona Center for Integrative Modeling \& Simulation (ACIMS). His research interests include model theory, poly-formalism modeling, collaborative modeling, simulation for complexity science, and M\&S frameworks/tools. He can be contacted at sarjoughian@asu.edu. 